\title{
Non-negative Matrix Factorization for Filtering Chinese Document*
}

\author{
Jianjiang $\mathrm{Lu}^{1,2,3}$, Baowen $\mathrm{Xu}^{1,2}$, Jixiang Jiang ${ }^{1}$, and Dazhou Kang ${ }^{1}$ \\ ${ }^{1}$ Department of Computer Science and Engineering, Southeast University, \\ Nanjing, 210096, China \\ ${ }^{2}$ Jiangsu Institute of Software Quality, Nanjing, 210096, China \\ ${ }^{3}$ PLA University of Science and Technology, Nanjing, 210007, China
}

jjlu@seu.edu.cn

\begin{abstract}
There are two nasty classical problems of synonymy and polysemy in the filtering systems of Chinese documents. To deal with these two problems, we would ideally like to represent documents not by words, but by the semantic relations between words. Non-negative matrix factorization (NMF) is applied to dimensionality reduction of the words space. NMF is distinguished from the latent semantic indexing (LSI) by its non-negativity constraints. These constraints lead to a parts-based representation because they allow only additive, not subtractive, combinations. Also, NMF computation is based on the simple iterative algorithm; it is therefore advantageous for applications involving large sparse matrices. The experimental results show that, comparing with LSI, NMF method not only improves filtering precision markedly, but also has the merits of fast computing speed and less memory occupancy.
\end{abstract}

\section{Introduction}

Automatic filtering of information from document sources has become increasingly important in recent years. Information filtering systems are designed to shift through large quantities of dynamically generated documents and display only those which may be relevant to a user's interests [1]. Two major types of filtering systems have been proposed: content-based filtering [2] and collaborative filtering. Collaborative filtering selects documents based on user's evaluations of the documents. On the other hand, content-based filtering selects documents based on the contents of documents and each user's preference.

There exist several types of content-based filtering systems. In the vector space model [3], user profiles and document profiles are represented as weighted vectors of the words in the system. The relevance of each document to each user is calculated according to the similarity between the user profile vector and the document profile vector. There are two nasty classical problems of synonymy and polysemy in the vector space model. To deal with these two and other similar problems, we would

\footnotetext{
This work was supported in part by the Young Scientist's Fund of NSFC (60373066, 60303024), National Grand Fundamental Research 973 Program of China (2002CB312000), National Research Foundation for the Doctoral Program of Higher Education of China.
} 
ideally like to represent documents not by words, but by the semantic relations between words.

Latent semantic indexing (LSI) analysis based on singular-value decomposition (SVD) $[4,5]$ is an information retrieval method that attempts to capture the semantic relations by using techniques from linear algebra. LSI constructs a low-dimensional semantic space wherein words and documents that are closely associated are placed near one another. SVD allows the arrangement of the space to reflect the major associative patterns in the data, and ignore the smaller, less important influences. However, the cost of SVD computation will be prohibitive when matrices become large. In addition, SVD is lack of intuitive notion.

In this paper, a method based on non-negative matrix factorization (NMF) [6, 7] for constructing Chinese user profile is presented. This method proposes to apply NMF to dimensionality reduction of the document vectors. NMF can decompose a non-negative matrix into two non-negative matrices. One of the decomposed matrices can be regarded as the basis vectors. The dimensionality reduction can be performed by projecting the document vectors onto the lower dimensional space which is formed by these basis vectors. NMF is distinguished from LSI by its non-negativity constraints. These constraints lead to a parts-based representation because they allow only additive, not subtractive, combinations. Also, NMF computation is based on the simple iterative algorithm; it is therefore advantageous for applications involving large sparse matrices.

The remainder of this paper is organized as follows. In section 2, we briefly review how to represent a set of unstructured Chinese documents as a vector space model. In section 3, we introduce non-negative matrix factorization. In section 4, a $\mathrm{NMF}$ method for constructing Chinese user profile is presented. In section 5, the experimental results of NMF method are compared with LSI. Finally, section 6 gives the conclusions.

\section{Vector Space Models for Documents}

Let $D_{1}=\left\{d_{1}^{1}, d_{2}^{1}, \cdots, d_{n_{1}}^{1}\right\}$ be a set of Chinese topic documents, let $D_{2}=\left\{d_{1}^{2}, d_{2}^{2}, \cdots, d_{n_{2}}^{2}\right\}$ be a set of non-topic documents, $n_{1}+n_{2}=n, D=D_{1} \cup D_{2}$. We briefly review how to represent a set of unstructured Chinese documents as a vector space model. The preprocessing is as following.

(1) Chinese documents are written as characters strings with no spaces between words, so we first use word segmentation algorithm [8] to segment the Chinese documents.

(2) After word segmentation, we eliminate non-content-bearing "stopwords".

(3) Using heuristic or information-theoretic criteria, eliminate non-content-bearing "high-frequency" and "low-frequency" words. Such words and the stopwords are both known as "function" words. Eliminating function words removes little or no information, while speeding up the computation.

(4) After above elimination, suppose $m$ unique words remain, let be $T=\left\{t_{1}, t_{2}, \cdots, t_{m}\right\}$. We use normalized word frequency-inverse document frequency 
scheme [9] to obtain word-document matrix $X=\left(x_{i j}\right)_{m \times n}$. The $i^{\text {th }}$ element $x_{i j}$ of the document vector $x_{j}=\left(x_{1 j}, x_{2 j}, \cdots, x_{m j}\right)^{T}$ is given by

$$
x_{i j}=\#\left(x_{j}, t_{i}\right) \log \frac{n}{h_{i}}
$$

where $\#\left(x_{j}, t_{i}\right)$ denotes the number that the word $t_{i}$ appears in the document $x_{j}, h_{i}$ denotes the number of the documents in which the word $t_{i}$ appears, $n$ is the total document number. Document vectors are usually normalized to a unit vector, that is,

$$
x_{i j}=\frac{x_{i j}}{\sqrt{\sum_{i=1}^{m} x_{i j}^{2}}}, i=1,2, \cdots, m
$$

Intuitively, the effect of normalization is to retain only the direction of the document vectors. This ensures that documents dealing with the same subject matter (that is, using similar words), but differing in length lead to similar document vectors.

After the preprocessing, Chinese documents are represented as $\mathrm{m}$ dimensional document vectors $x_{j}, j=1,2, \cdots, n$. Let $X_{1}=\left\{x_{1}^{1}, x_{2}^{1}, \cdots, x_{n_{1}}^{1}\right\}$ be topic document vectors, $X_{2}=\left\{v_{1}^{2}, v_{2}^{2}, \cdots, v_{n_{2}}^{2}\right\}$ be non-topic document vectors, $X=X_{1} \cup X_{2}$. These document vectors make up of word-document matrix $X=\left(x_{i j}\right)_{m \times n}$.

\section{Non-negative Matrix Factorization}

Given a non-negative matrix $X=\left(x_{i j}\right)_{m \times n}$, NMF finds the non-negative $m \times r$ matrix $U=\left(u_{i j}\right)_{m \times r}$ and the non-negative $r \times n$ matrix $V=\left(v_{i j}\right)_{r \times n}$ such that

$$
X \approx U V
$$

The $r$ is generally chosen to satisfy $(n+m) r<n m$, so that the product $U V$ can be regarded as a compressed form of the data in $X$.

The equation (1) can be rewritten column by columns as

$$
x \approx U v
$$

where $x$ and $v$ are the corresponding columns of $X$ and $V$. Each vector $x$ is approximated by a linear combination of the columns of $U$, weighted by the components of $v$. Therefore, $U$ can be regarded as containing a basis vector that is optimized for the linear approximation of the vector in $X$. Since relatively few basis vectors are used to represent many vectors, good approximation can only be achieved if the basis vectors discover structure that is latent in the vectors.

Here, we introduce an algorithm based on iterative estimation of $U$ and $V$. At each iteration of the algorithm, the new value of $U$ and $V$ is found by multiplying the current value by some factor that depends on the quality of the approximation in equation (1). Repeated iteration of the update rules is guaranteed to converge to a locally optimal matrix factorization.

The update rules given in the next equations [7]. 


$$
\begin{gathered}
v_{i j} \leftarrow \sqrt{v_{i j} \sum_{k} u_{k i} \frac{x_{k i}}{y_{k j}}} \\
u_{i j} \leftarrow u_{i j} \sum_{k} \frac{x_{i k}}{y_{i k}} v_{j k} \\
u_{i j} \leftarrow \frac{u_{i j}}{\sum_{k} u_{k j}}
\end{gathered}
$$

where $U$ and $V$ are initial stochastic matrices.

The update rules maximize the following objective function:

$$
F(X, Y)=\sum_{i, j}\left(x_{i j} \log \frac{x_{i j}}{y_{i j}}-x_{i j}+y_{i j}\right)+\alpha \sum_{i, j} a_{i j}-\beta \sum_{i} b_{i i}
$$

where $a_{i j}$ is the components of $U^{T} U, b_{i i}$ is the diagonal components of $V V^{T}, \alpha, \beta>0$ are some constants, $Y=U V=\left(y_{i j}\right)_{m \times n}$.

\section{Constructing Chinese User Profile}

After word-document matrix $X$ is decomposed by the NMF in section 3, the $m$ dimensional document vectors are projected into the $r$ dimensional vectors. Let $V_{1}=\left\{v_{1}^{1}, v_{2}^{1}, \cdots, v_{n_{1}}^{1}\right\}$ be projecting of the topic document vectors, $V_{2}=\left\{v_{1}^{2}, v_{2}^{2}, \cdots, v_{n_{2}}^{2}\right\}$ be projecting of the non-topic document vectors. We can compute the mean vector of topic document vectors.

$$
O=\left(o_{1}, o_{2}, \cdots, o_{m}\right)=\frac{1}{n_{1}} \sum_{j=1}^{n_{1}} x_{j}^{1}
$$

In the same way, we can compute the mean vector of projecting of the topic document vectors.

$$
O^{1}=\left(o_{1}^{1}, o_{2}^{1}, \cdots, o_{r}^{1}\right)=\frac{1}{n_{1}} \sum_{j=1}^{n_{1}} v_{j}^{1}
$$

According to equation (2), we can easily obtain.

$$
O \approx U O^{1}=u_{1} o_{1}^{1}+u_{2} o_{2}^{1}+\cdots+u_{r} o_{r}^{1}
$$

A simply way is to select $U O^{1}$ as a user profile, but this user profile may be ineffective. Next, we define the class discriminative degree of the basis vectors in order to obtain an effective user profile. 
Definition1. Let $v_{j}^{1}=\left(v_{1 j}^{1}, v_{2 j}^{1}, \cdots, v_{r j}^{1}\right)^{T}, \quad j=1,2, \cdots, n_{1}, \quad v_{j}^{2}=\left(v_{1 j}^{2}, v_{2 j}^{2}, \cdots, v_{r j}^{2}\right)^{T}$, $j=1,2, \cdots, n_{2}$ Class discriminative degree of the basis vector $u_{s}$ to topic documents is defined as follows:

$$
d_{s}=\frac{1}{n_{1}} \sum_{j=1}^{n_{1}} v_{s j}^{1}-\frac{1}{n_{2}} \sum_{j=1}^{n_{2}} v_{s j}^{2}, s=1,2, \cdots, r
$$

If the average weight $\frac{1}{n_{1}} \sum_{j=1}^{n_{1}} v_{s j}^{1}$ of the basis vector $u_{s}$ in the topic documents is big, and the average weight $\frac{1}{n_{2}} \sum_{j=1}^{n_{2}} v_{s j}^{2}$ of the basis vector $u_{s}$ in the non-topic documents is small, then the class discriminative degree $d_{s}$ is large. That is to say, the basis vector $u_{s}$ has strong discriminative ability between topic documents and the non-topic documents. We select $k$ basis vectors with big class discriminative degrees, simply let be $u_{1}, u_{2}, \cdots, u_{k}, k \leq r$. According to equation (7), we use these $\mathrm{k}$ basis vectors to construct a $m$ dimensional vector of the words as follows.

$$
u_{1} o_{1}^{1}+u_{2} o_{2}^{1}+\cdots+u_{k} o_{k}^{1}
$$

Then, we sort the components of the $m$ dimensional vector by the value, and select $l$ components with big values as the user profile.

$$
\text { User_Profile } \left.\left.\left.=\left\{<t_{1}, g_{1}\right\rangle,<t_{2}, g_{2}\right\rangle, \cdots,<t_{l}, g_{l}\right\rangle\right\}
$$

where $t_{i}$ is a word, $g_{i}$ is the component value with respect to word $t_{i}, i=1,2, \cdots, l$.

\section{Experimental Results}

All documents in the experiment are downloaded from http://www.sina.com.cn. The topic documents include: Chess (277), Gym (149), Badminton (110), Box (69), PingPong (177), Volleyball (171), Racing (95), Swimming (126), Tennis ball (208), Baseball (60), Skating (155), Golf (111), Track and field (147), Billiards (58) and Martial art (43). In addition, there have 2044 non-topic documents.

In order to test 15 topic documents, we partition each topic documents into four groups, and select one group as testing documents, other groups as training documents. When recall was set to $0 \%, 10 \%, 20 \%, 30 \%, \ldots, 90 \%, 100 \%$, the average precision at 11 points of filtering systems based on NMF and LSI [5] are compared.

We select 2843 words in the experiment, let $r=200$, figure 1 shows the experimental results on the four topic documents. Figure 1(a) and figure 1(b) show the average precisions of filtering systems based on NMF and LSI with the selecting words respectively. In the filtering systems based on NMF, we select $\mathrm{k}$ basis vectors that have strong discriminative ability between topic documents and the non-topic documents. Figure 2 shows the average precisions of 15 topic documents.

The experimental results show that the average precision of filtering systems based on NMF is better than LSI. In addition, the memory occupancy of NMF is lesser than LSI. For example, the memory occupancy of the left singular matrix in LSI is 
$126.3 \mathrm{M}$, and the memory occupancy of the right singular matrix is $33.0 \mathrm{M}$. Whereas the memory occupancy of the left non-negative matrix in NMF is $6.9 \mathrm{M}$, and the memory occupancy of the right non-negative matrix is $6.7 \mathrm{M}$. Furthermore, NMF only needs 30-40 iterative times, so NMF costs less computation time than LSI. In the experiment, using the SVDPACK from http://www.netlib.org/svdpack, NMF only costs about half time of LSI.

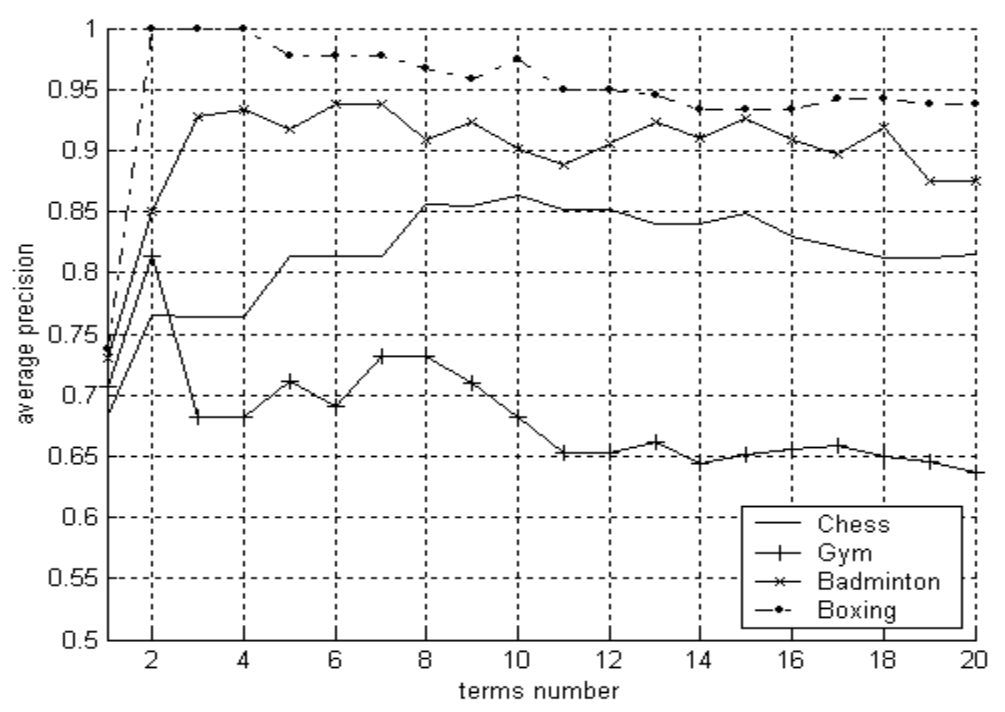

(a) LSI ( $r=200)$

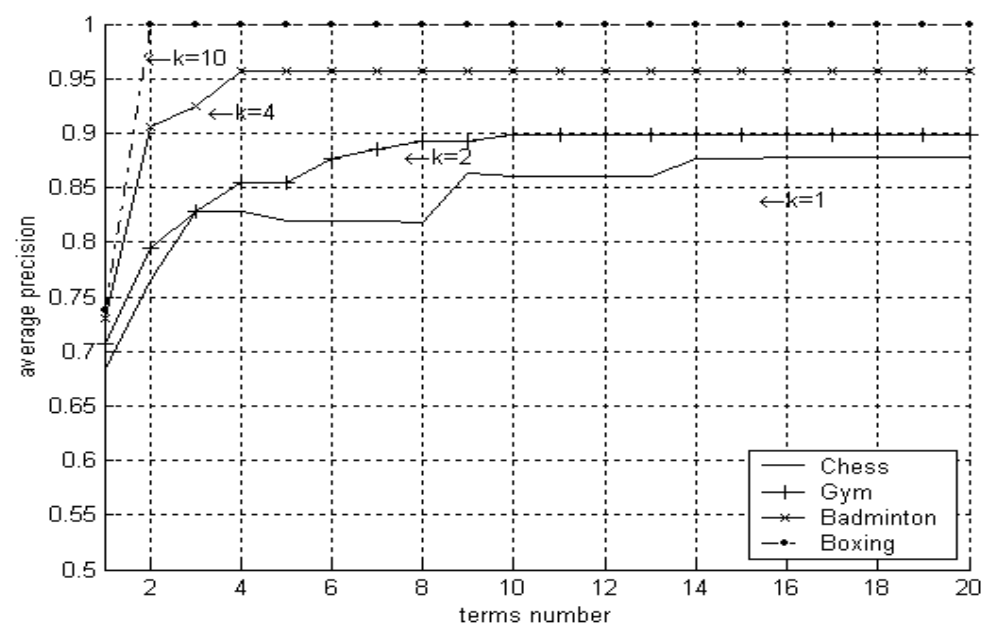

(b) NMF ( $\mathrm{r}=200)$

Fig. 1. Comparing the precision 


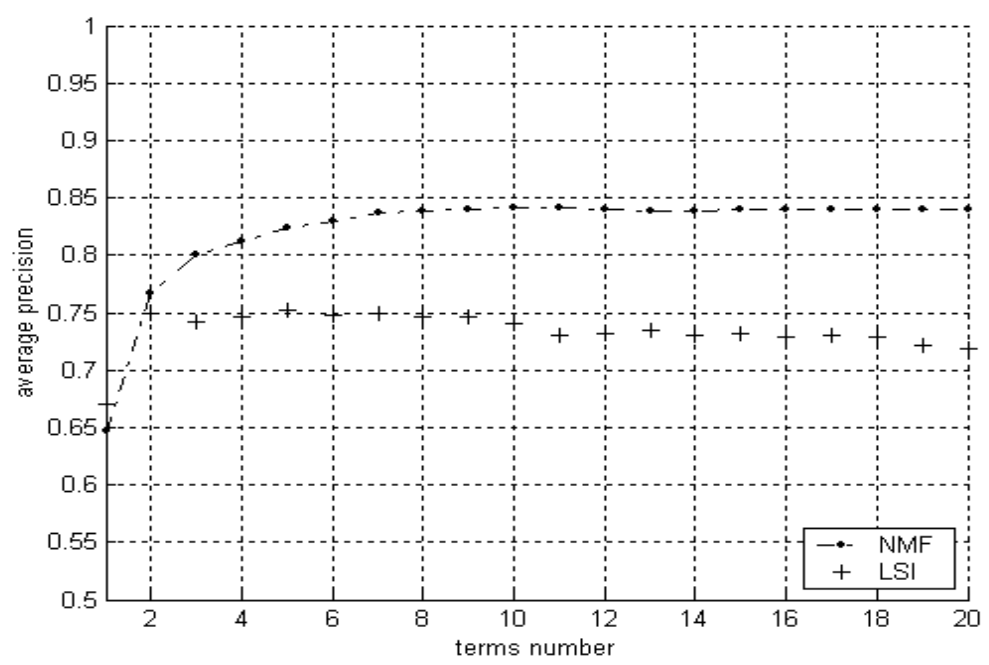

Fig. 2. Comparing average precision

\section{Conclusion}

Automatic filtering of information from document sources has become increasingly important as the volume of electronically accessible documents has exploded in recent years. In this paper, a method based on NMF for constructing Chinese user profile is presented. This method proposes to apply NMF to dimensionality reduction of the document vectors in the word-document matrices. NMF decomposes a non-negative matrix into two non-negative matrices. One of the decomposed matrices can be regarded as the basis vectors. The dimensionality reduction can be performed by projecting the document vectors onto the lower dimensional space which is formed by these basis vectors. NMF is distinguished from LSI by its non-negativity constraints. These constraints lead to a parts-based representation because they allow only additive, not subtractive, combinations. Also, NMF computation is based on the simple iterative algorithm, it is therefore advantageous for applications involving large sparse matrices. The experimental results show that, comparing with LSI, NMF method not only improves filtering precision markedly, but also has the merits of fast computing speed and less memory occupancy. In the future work, we will discuss how to use NMF in the Chinese document clustering and classification. 


\section{References}

1. Belkin, N.J., Croft, W. B.: Information Filtering and Information Retrieval: two sides of the same coin. Communication of ACM, 35(12). (1992) 29-38

2. Chen, L., Katia, S.: WebMate: A Personal Agent for Browsing and Searching. ACM AGENTS'98, Proceedings of the International Conference on Autonomous Agents, Minneapolis (1998) 132-139

3. Yart, T. W., Garcia-Molina, H.: Index Structures for Information Filtering under the Vector Space Model. Proceedings of the 10th International Conference on Data Engineering, Alamitos, CA, IEEE (1994) 337-347

4. Papadimitriou, C. H., Raghavan, P., Tamaki, H.: Latent Semantic Indexing: A Probabilistic Analysis. Proceedings of PODS'98, Seattle, WA (1998)159-168

5. Lu, Z., Lu, H., Li, Y.: FDS Expressive Method in Information Filtering. Journal of Tsinghua University (science and technology), 39(9). (1999)118-121

6. Lee, D. D., Seung, H. S.: Learning the Parts of Objects by Non-negative Matrix Factorization. Nature, 401. (1999) 788-791

7. Li, S. Z., Hou, X. W., Zhang, H. J.: Learning Spatially Localized Parts-based Representation. Proceedings of IEEE International Conference on Computer Vision and Pattern Recognition, Hawaii (2001) 207 -212

8. Chen, G. L., Wang, Y. C., HAN, K. S., Wang, G.: An Improved Fast Algorithm for Chinese Word Segmentation. Journal of Computer Research and Development, 37(4). (2000) 418-424

9. Kolda, T. G.: Limited-Memory Matrix Methods with Applications. Ph.D. thesis, The Applied Mathematics Program, University of Maryland, College Park, Mayland (1977) 